\title{
Engagement y optimismo en un grupo de trabajadores colombianos: análisis de algunos predictores
}

\author{
Engagement and optimism in a group of Colombian workers: analysis of some predictors
}

\author{
Luisa Fernanda Ocampo-Álvarez ${ }^{1}$ orcid.org/0000-0002-4697-1430 \\ Eliana Quiroz-González1* orcid.org/0000-0001-9061-8864 \\ Erika Villavicencio-Ayub² orcid.org/0000-0003-0021-5020
}

1. Programa de Psicología. Universidad Católica de Pereira. Pereira, Colombia.

2. Facultad de Psicología. Universidad Nacional Autónoma de México. Ciudad de México, México.

Ocampo-Álvarez LF, Quiroz-González E, Villavicencio-Ayub E. Engagement y optimismo en un grupo de trabajadores colombianos: análisis de algunos predictores. Univ. Salud. Suplemento1: Especial Psicología y Trabajo. 2021; 23(3):320-328. DOI: https://doi.org/10.22267/rus.212303.246

\section{Resumen}

Introducción: El engagement y el optimismo aportan a la salud mental, por tanto, es importante avanzar en la investigación de estos fenómenos psicológicos poco estudiados en Colombia. Objetivo: Analizar la relación entre el engagement y el optimismo en un grupo de trabajadores colombianos, e identificar el papel predictivo de la edad y la antigüedad en la organización en el engagement y el optimismo. Materiales y métodos: Se utilizó una estrategia asociativa donde participaron 298 trabajadores $(\mathrm{M}=124-\mathrm{H}=174)$ de una organización del sector telecomunicaciones. Se aplicó un cuestionario sociodemográfico, el Utrecht Work Engagement Scale-17 y el Life Orientation Test. Las hipótesis se probaron mediante correlación de Pearson, análisis de varianza y modelo de regresión lineal múltiple de pasos sucesivos. Resultados: Las dimensiones del engagement se correlacionaron con el optimismo vigor $(\mathrm{r}=0,42, p<0,01)$, dedicación $(\mathrm{r}=0,26, p<0,01)$ y absorción $(\mathrm{r}=0,20, p<0,01)$ ). Conjuntamente, se encontraron diferencias en la dedicación en función de la edad $(p=0,01)$ y la antigüedad $(p=0,04)$. Los modelos predictivos reportaron asociaciones entre edad y vigor $(\beta=0,25, \mathrm{t}=4,2, p<0,000)$, dedicación $(\beta=0,202, \mathrm{t}=3,38, p<0,001) \mathrm{y}$ absorción $(\beta=0,145, \mathrm{t}=2,4$, $p<0,017)$. Conclusiones: Existe una relación positiva entre engagement y optimismo. En este estudio la edad es un predictor del engagement, pero no del optimismo.

Palabras clave: Indicadores demográficos; optimismo; salud laboral; salud mental; rendimiento laboral, compromiso. (Fuente: DeCS, Bireme).

\begin{abstract}
Introduction: Engagement and optimism contribute to mental health. Therefore, it is important to carry out research on these psychological phenomena that are poorly studied in Colombia. Objective: To analyze the relationship between engagement and optimism in a group of Colombian workers and identify the role of age and job seniority in predicting these two phenomena. Materials and methods: An associative strategy was applied, with the participation of 298 workers (124 men and 174 women) from a Telecommunications company. The Utrecht Work Engagement Scale-17 and Life Orientation sociodemographic questionnaires were used. The hypothesis was tested through Pearson correlation, variance analysis, and multiple linear regression model with successive steps. Results: The engagement dimensions correlated with optimism (vigor ( $\mathrm{r}=0.42, p<0.01)$, commitment $(\mathrm{r}=0.26, p<0.01)$ and absorption $(\mathrm{r}=0.20, p<0.01))$. Overall, there were differences between commitment in reference to age $(p=0.01)$ and seniority $(p=0.04)$. Predictive models showed associations between age and vigor $(\beta=0.25, \mathrm{t}=4.2, p<0.000)$, commitment $(\beta=0.202, \mathrm{t}=3.38, p<0.001)$ and absorption $(\beta=0.145, \mathrm{t}=2.4, p<0.017)$. Conclusions: There is a positive relationship between engagement and optimism. Based on this study, even though age is a predictor of engagement, it does not play the same role for optimism.
\end{abstract}

Keywords: Demographic indicators; optimism; occupational health; mental health; labor performance, commitment. (Source: DeCS, Bireme). 


\section{Introducción}

Las condiciones actuales en los mercados laborales llevan a que las organizaciones adopten enfoques competitivos en la gestión del talento humano que promuevan el cumplimiento de los índices de productividad, y a la par cuenten con una fuerza de trabajo con índices positivos en satisfacción, lo que implica otorgar un lugar central al bienestar y el desarrollo humano en los contextos empresariales(1). Para ello, las organizaciones deben centrar su atención en la motivación, las habilidades, el desempeño y las relaciones positivas en sus equipos de trabajo, pues esto permite al empleado afrontar las exigencias que se le presenten en su vida diaria, al tiempo que facilita que las instituciones mejoren su efectividad(2,3), cuenten con fortalezas financieras y sean rentables en un entorno que se caracteriza por ser cambiante(4). Es aquí donde juegan un papel importante el engagement y el optimismo, los cuales favorecen el bienestar físico, psicológico y la calidad de vida laboral(2).

El engagement es un estado cognitivo-afectivo que perdura en el tiempo y se focaliza en el trabajo, este constructo motivacional está compuesto por el vigor, la dedicación y la absorción. El vigor implica la energía con la que el trabajador lleva a cabo sus funciones, su activación mental y deseo por invertir esfuerzo en la tarea; la dedicación hace referencia a estar fuertemente involucrado con el trabajo, experimentando altos niveles de significado, entusiasmo, orgullo e inspiración; la absorción está asociada a la identificación, concentración y disfrute en el trabajo. El trabajador engaged encuentra desafíos en su trabajo, disfruta la tarea y siente que el tiempo pasa rápido mientras trabaja(2). Además, el engagement aporta al cumplimiento de los objetivos organizacionales(5), tiene implicaciones positivas en el clima organizacional y se relaciona con la iniciativa personal, la autoeficacia y el desempeño(6,7).

El engagement junto con el optimismo son atributos personales positivos que están relacionados con la capacidad para hacerle frente a acontecimientos estresantes en la vida diaria(8). Con ello, diferentes estudios internacionales reportan una relación positiva entre estas dos variables ${ }^{(9,10)}$. En este orden de ideas, el optimismo disposicional es un sistema de creencias y expectativas que tienen las personas acerca de los resultados positivos futuros, los cuales están relacionados con la motivación(11). Las personas optimistas se ajustan de manera favorable a los cambios organizacionales(12), tienden a ser más autoeficaces $^{(9,13)}$ y felices(14), realizan más esfuerzos para conseguir un propósito en comparación con los pesimistas(11) y tienen un papel protector frente a las consecuencias del estrés(15).

Dada la relevancia del engagement y el optimismo en la salud y la gestión organizacional(2), es importante estudiar la relación entre ellas y avanzar en la comprensión de estos fenómenos psicosociales positivos, específicamente en un contexto latinoamericano, y así, contribuir desde la investigación en la consolidación de organizaciones saludables. Cabe resaltar que este tipo de organizaciones generan beneficios bidireccionales, es decir, para la organización y para los trabajadores ${ }^{(4)}$.

Por lo anterior, este estudio propone analizar la relación entre el engagement y el optimismo en un grupo de trabajadores colombianos del sector de las telecomunicaciones, de forma que se realice un aporte basado en la evidencia, que oriente a los tomadores de decisiones en las organizaciones sobre posibles vías para promover la salud mental de sus trabajadores. Asimismo, se busca identificar el papel predictivo de la edad y la antigüedad en la organización en el engagement y el optimismo, dado que, pese a que algunos reportes indican que estas variables sociodemográficas no se relacionan o diferencian ni con el engagement(16) ni con el optimismo(10,17,18), otros han encontrado que los empleados que tienen más antigüedad en la organización, poseen mayor engagement(19) y que las personas de mayor edad son más optimistas(20).

\section{Materiales y métodos}

\section{Diseño y participantes}

Estudio con una estrategia asociativa, con diseño predictivo, transversal(21). Se realizó un muestreo no probabilístico, a conveniencia, en el que participaron 298 trabajadores de una organización colombiana del sector de las telecomunicaciones con sede en varios países de América Latina; en Colombia esta organización está conformada por un total de 394 trabajadores. Como criterio de inclusión, los participantes debían contar con más de seis meses de vinculación a la organización. 


\section{Instrumentos}

La información sobre la edad y antigüedad en la organización se obtuvo a través de un cuestionario de datos sociodemográficos ad hoc. Para medir el engagement se utilizó el Utrecht Work Engagement Scale - 17, en su adaptación colombiana ${ }^{(22)}$, con el cual se obtuvo un índice de confiabilidad de 0,902. Este instrumento está compuesto por 17 ítems, en donde se evalúan las tres dimensiones: vigor, dedicación y absorción, por medio de una escala tipo Likert de 7 opciones de respuesta entre 1 (Nunca) y 7 (Siempre). Se estimó la consistencia interna en este estudio a partir del Omega de McDonald's $\omega$ obteniendo los siguientes resultados por dimensión: vigor: 0,84, dedicación: 0,87 y absorción: 0,78 , toda la escala tuvo una puntuación de 0,93.

Para evaluar el optimismo se utilizó el Life Orientation Test en su versión revisada(23). Este instrumento consta de 10 ítems, cuatro de ellos son de relleno, tres están en dirección negativa y tres en sentido positivo. $\mathrm{Su}$ escala es tipo Likert, con cinco opciones de respuesta entre 0 (muy en desacuerdo) y cuatro (muy de acuerdo). Un estudio previo en Colombia comprueba su calidad psicométrica(24). Para este estudio, el Omega de McDonald's $\omega$ fue de 0,63.

\section{Procedimiento}

El proyecto fue aprobado por los directivos de la organización, posteriormente, se envió el consentimiento informado a los trabajadores, seguido de los cuestionarios a través de un formulario de Google ${ }^{\circledR}$. Los participantes se agruparon en dos grupos de acuerdo con la edad (G1: 18 a 30 años - G2: 31 a 61 años) y en 3 grupos en función de la antigüedad en la organización (G1: 6 a 24 meses, G2: 36 a 48 meses y G3: 60 a 278 meses). La recolección de los datos se llevó a cabo durante el segundo semestre del año 2019.

\section{Análisis de datos}

Se diseñó una matriz en Excel para ordenar y agrupar los datos que posteriormente se exportaron al paquete estadístico SPSS versión 22 para calcular medidas de tendencia central (M), dispersión (DE) y las correlaciones a partir de la técnica productomomento de Pearson. Se realizó un análisis diferencial para comparar el engagement y el optimismo con dos grupos de edad (G1: 18 a 30 años, G2:31 a 61 años), así como un análisis de varianza para identificar la diferencia en el engagement y el optimismo en función de la antigüedad en la organización desde tres grupos etarios (G1: 6 a 24 meses, G2: 36 a 48 meses y G3: 60 a 278 meses). La confiabilidad se midió con el coeficiente Omega de McDonald's $\omega$ usando el software Jamovi ${ }^{\circledR}$. Se asumió un valor $p<0,05$.

Igualmente, se realizó un análisis multivariante mediante la técnica de regresión lineal múltiple de pasos sucesivos para determinar la influencia de las variables independientes (edad y antigüedad en la organización) sobre las dependientes (engagement y optimismo), posterior al cumplimiento de los supuestos de multicolinealidad, normalidad e independencia de errores; consecutivamente, se ejecutó un análisis de la varianza (ANOVA o AVAR) para determinar el ajuste de cada modelo, estableciendo para cada variable un nivel de significación: $\alpha=0,05$.

\section{Consideraciones éticas}

Este estudio se acoge a las disposiciones de la Ley 1090 del 2006(25) y la Resolución 8430 de 1993(26). Los trabajadores aceptaron su participación en esta investigación a través de la aprobación del consentimiento informado, el cual se presentó de manera previa a las escalas en el formulario de Google ${ }^{\circledR}$. La participación se dio de manera voluntaria y se conservó la confidencialidad de los datos. El proyecto investigativo $y$ el formato de consentimiento informado fue avalado por el Comité de Ética de la Universidad Católica de Pereira como consta en el Acta No. 002 del 08 de agosto de 2019.

\section{Resultados}

De los participantes, 174 eran hombres (58\%) y 124 mujeres (42\%), 153 (51\%) estaban entre los 18 y 30 años, 105 (35\%) entre los 31 y 40 años, 32 (11\%) entre los 41 y 51 años y 8 participantes (3\%) tenían entre 51 y 61 años. Además, 151 (51\%) tenía entre 6 meses y menos de 3 años de antigüedad en la organización, 84 (28\%) entre 3 y menos de 6 años, 40 (13\%) entre 6 y menos de 9 años y 23 (8\%) con más de 9 años. La mayoría de los trabajadores contaban con un contrato a término indefinido $(\mathrm{N}=239-80 \%)$, tenían formación técnica ( $\mathrm{N}=145-49 \%)$ y estaban solteros ( $\mathrm{N}=136-46 \%)$.

El resultado del análisis de correlación (Tabla 1) indica que las tres dimensiones del engagement se correlacionaron positivamente con el optimismo, de 
éstas, la relación más fuerte se encuentra entre vigor y optimismo, además las dimensiones del engagement y optimismo correlacionan con la edad a un nivel de $p<0,01$, sobre todo la dimensión de vigor $(r=0,24, p<0,01)$. Por otro lado, no existe relación entre la antigüedad en la organización con las dimensiones del engagement, aunque la antigüedad en la organización se relacionó muy débil con el optimismo de los trabajadores $(r=0,12, p<0,05)$. Por último, se identifican correlaciones altas $y$ significativas entre las dimensiones del engagement, resultado esperado a nivel teórico.

Para analizar la variable edad con las variables en estudio se realiza la comparación de medias con una $\mathrm{T}$ de Student. Las variables de vigor, absorción y optimismo no reportan diferencias estadísticamente significativas en función de la edad, mientras que la dedicación se diferencia significativamente (Tabla 2).
$\mathrm{Al}$ contrastar los tres grupos en torno a la antigüedad en la organización, el análisis de varianza indica que para el vigor y absorción no existen diferencias significativas, pero la variable dedicación reporta diferencias, el contraste intergrupo a través de la prueba Tukey indicó diferencias entre grupo $1 \mathrm{y}$ grupo $2(p=0,003)$ (Tabla 3$)$.

Con el fin de determinar si la antigüedad en la organización y la edad predicen las dimensiones del engagement, se realizó un análisis de regresión múltiple. Se utilizó el método de pasos sucesivos debido a que el análisis de correlación indicó que existe una relación positiva y directa entre la mayoría de las variables. El análisis de pasos sucesivos identifica el mejor predictor de la variable dependiente, seleccionando aquel con la correlación más alta, si ese predictor mejora el modelo, este se mantiene y se busca un segundo predictor(27).

Tabla 1. Correlación del engagement y optimismo con las variables independientes

\begin{tabular}{|c|c|c|c|c|c|c|c|}
\hline Variable & M & DE & 1 & 2 & 3 & 4 & 5 \\
\hline \multicolumn{8}{|l|}{ Variable independiente } \\
\hline Edad & 31,62 & 7,97 & - & & & & \\
\hline Antigüedad en la organización & 49,96 & 39,60 & $0,29^{* *}$ & & & & \\
\hline \multicolumn{8}{|l|}{ Engagement } \\
\hline Vigor & 4,62 & 0,98 & $0,24^{* *}$ & 0,05 & - & & \\
\hline Dedicación & 4,91 & 1,06 & $0,19^{* *}$ & 0,01 & $0,80^{* *}$ & - & \\
\hline Absorción & 4,52 & 0,95 & $0,13^{* *}$ & 0,01 & $0,77^{* *}$ & $0,80^{* *}$ & - \\
\hline Optimismo & 17,69 & 3,39 & $0,21^{* *}$ & $0,12^{*}$ & $0,42^{* *}$ & $0,26^{* *}$ & $0,20^{* *}$ \\
\hline
\end{tabular}

Nota: ${ }^{* *} p<0,01,{ }^{*} p<0,05.1$ = Edad, $2=$ Antigüedad en la organización evaluada en meses, 3=Vigor, 4= Dedicación, 5= Absorción, 6= 0ptimismo.

Tabla 2. Comparación de la edad en función al engagement y el optimismo

\begin{tabular}{|c|c|c|c|c|c|c|}
\hline \multirow{2}{*}{ Variable } & \multirow{2}{*}{$\begin{array}{c}\text { G1 }(n=109) \\
M \pm D E\end{array}$} & \multirow{2}{*}{$\begin{array}{c}\text { G2 }(n=189) \\
M \pm D E\end{array}$} & \multirow[b]{2}{*}{$t$} & \multirow[b]{2}{*}{$P$} & \multicolumn{2}{|c|}{ 95\% IC } \\
\hline & & & & & LI & LS \\
\hline Vigor & $4,7 \pm 0,96$ & $4,5 \pm 0,99$ & 1,84 & 0,67 & $-0,14$ & 0,44 \\
\hline Dedicación & $5,1 \pm 0,98$ & $4,7 \pm 1,08$ & 2,54 & 0,01 & 0,07 & 0,57 \\
\hline Absorción & $4,6 \pm 0,93$ & $4,4 \pm 0,95$ & 1,91 & 0,06 & $-0,00$ & 0,44 \\
\hline Optimismo & $17,6 \pm 3,3$ & $17,8 \pm 3,4$ & $-0,37$ & 0,70 & $-0,96$ & 0,65 \\
\hline
\end{tabular}

Nota: G1= grupo etario 1, 18 a 30 años; G2= grupo etario 2, 31 a 51 años.

Tabla 3. Análisis de varianza según antigüedad en el engagement y el optimismo

\begin{tabular}{|c|c|c|c|c|c|c|c|c|c|c|c|}
\hline \multirow{2}{*}{ Variable } & \multicolumn{3}{|c|}{ G1 $(n=109)$} & \multicolumn{3}{|c|}{ G2 (n=83) } & \multicolumn{3}{|c|}{ G3 $(n=106)$} & \multirow{2}{*}{$\mathbf{F}$} & \multirow{2}{*}{$P$} \\
\hline & $M$ & \pm & $D E$ & $M$ & \pm & $D E$ & $M$ & \pm & $D E$ & & \\
\hline Vigor & 4,7 & \pm & 0,96 & 4,4 & \pm & 0,92 & 4,6 & \pm & 1,03 & 2,817 & 0,061 \\
\hline \multirow[t]{2}{*}{ Dedicación } & 5,1 & \pm & 0,98 & 4,6 & \pm & 1,13 & 4,9 & \pm & 1,02 & 5,684 & 0,004 \\
\hline & & G1 & & & G2 & & & G3 & & & 0,003 \\
\hline Absorción & 4,6 & \pm & 0,93 & 4,3 & \pm & 0,94 & 4,5 & \pm & 0,96 & 2,463 & 0,087 \\
\hline Optimismo & 17,6 & \pm & 3,3 & 17,2 & \pm & 3,5 & 18,2 & \pm & 3,3 & 1,839 & 0,161 \\
\hline
\end{tabular}

Nota: G1= grupo 1, 6 a 24 meses; G2= grupo 2, 36 a 48 meses; G3= grupo 3, 60 a278 meses. 
El análisis de regresión está diseñado bajo tres modelos: cada uno incluye diferentes variables dependientes (vigor, dedicación, absorción y optimismo) y mantiene fijo en cada modelo los predictores (edad y antigüedad en la organización). El resultado del análisis indica que, en el primer modelo, el valor de $R^{2}$ explica un $15,6 \%$ de la variación mientras que en el segundo el 19,3\%. En el caso de la significancia de los modelos, ambos resultan ser significativos con valores de $p=0,000$ para el primer modelo y $p=0,000$ para el segundo (Tabla 4). El cambio en el valor de $F$ significa la diferencia que se obtiene cuando se agregan nuevos predictores al modelo(27).

Como se observa en la Tabla 4, sólo la edad se relacionó significativamente con el vigor $(\beta=0,25$, $\mathrm{t}=4,2, p<0,000$ ) y representa el $5,9 \%$ de la varianza con la dimensión vigor $\left(\mathrm{R}^{2}=0,059, \mathrm{~F}=18,52, p<0,01\right)$. En cambio, la antigüedad en la organización se relacionó negativamente con el vigor, pero no de manera significativa $(\beta=-0,043, t=-0,717, p<0,474)$. En el segundo modelo correspondiente a la dedicación, la edad se relaciona significativamente con la dedicación $(\beta=0,202, \mathrm{t}=3,38, \quad p<0,001) \mathrm{y}$ representa el $3,6 \%$ de la varianza con la dimensión dedicación $\left(\mathrm{R}^{2}=0,036, \mathrm{~F}=11,00, p<0,01\right)$. Por otra parte, la antigüedad en la organización se relacionó negativamente con la dedicación, pero no de forma significativa $(\beta=-0,043, \mathrm{t}=-0,717, p<0,474)$.

En cuanto a la última dimensión del engagement, la edad se relacionó significativamente con la absorción $(\beta=0,145, t=2,4, p<0,017)$ y explica el $1,8 \%$ de la varianza en la dimensión absorción $\left(\mathrm{R}^{2}=0,018\right.$, $\mathrm{F}=5,54, p<0,01)$; la antigüedad en la organización reportó la misma tendencia estadística que tuvo con las demás dimensiones de engagement.

Finalmente, la edad no se relacionó significativamente con el optimismo $(\beta=0,109$, $\mathrm{t}=1,81, p>0,071)$ y representó el $2,5 \%$ de la varianza del optimismo $\left(\mathrm{R}^{2}=0,025, \mathrm{~F}=5,42, p>0,05\right)$. Lo anterior indica que la edad de los trabajadores no predice el optimismo. Por otro lado, la antigüedad en la organización no aportó significativamente al modelo de optimismo $(\beta=0,089, \mathrm{t}=1,48, p>0,140)$.

Tabla 4. Regresión entre las dimensiones del engagement, la edad y la antigüedad en la organización

\begin{tabular}{|c|c|c|c|c|c|c|c|}
\hline & $B$ & $\mathbf{B}$ & $\mathbf{T}$ & $P$-valor & $\mathbf{R}^{2}$ & $R^{2}$ ajustado & $\mathbf{F}$ \\
\hline Modelo con vigor & & & & & 0,059 & 0,056 & $18,52^{* *}$ \\
\hline Constante & 3,68 & & 16,17 & 0,000 & & & \\
\hline Edad & 0,031 & 0,25 & 4,2 & 0,000 & & & \\
\hline Antigüedad en organización & $-0,001$ & $-0,024$ & $-0,404$ & 0,687 & & & \\
\hline Modelo con dedicación & & & & & 0,036 & 0,033 & $11,00^{* *}$ \\
\hline Constante & 4,11 & & 16,62 & 0,000 & & & \\
\hline Edad & 0,027 & 0,202 & 3,38 & 0,001 & & & \\
\hline $\begin{array}{l}\text { Antigüedad en la } \\
\text { Organización }\end{array}$ & $-0,001$ & $-0,043$ & $-0,717$ & 0,474 & & & \\
\hline Modelo con absorción & & & & & 0,018 & 0,015 & $5,54^{* *}$ \\
\hline Constante & 4,01 & & 17,86 & 0,000 & & & \\
\hline Edad & 0,017 & 0,145 & 2,4 & 0,017 & & & \\
\hline Antigüedad en organización & $-0,001$ & $-0,32$ & -531 & 0,596 & & & \\
\hline
\end{tabular}

Nota: ${ }^{* *} p<0,01$.

\section{Discusión}

En el ámbito empresarial, el engagement es una de las variables que ha adquirido un importante interés(28,29), en coherencia con esto, una de las reflexiones está dirigida a la necesidad de documentar mayor evidencia empírica sobre los beneficios y relaciones con otras variables positivas ${ }^{(30)}$ en la gestión de culturas de bienestar(31), para lo cual cobra relevancia la asociación con el optimismo, el cual está vinculado al bienestar a lo largo del ciclo vital(32).

Los resultados obtenidos muestran una relación significativa entre estos dos constructos, lo cual coincide con lo hallado en otros estudios $(9,10,33,34)$, de manera que, a mayor vigor, dedicación y absorción, mayor optimismo en los trabajadores. Cabe 
mencionar que de las tres dimensiones del engagement, la relación más fuerte se encontró entre el vigor y el optimismo, hallazgos similares reportados previamente ${ }^{(9)}$. El vigor hace alusión a la energía y esfuerzo mental durante la ejecución de la tarea, por su parte, el optimismo se relaciona con las creencias positivas acerca de los resultados futuros. Partiendo de estos hallazgos, se asume que entre mayor sea la activación en los trabajadores mientras desempeñan sus labores, mayores expectativas positivas experimentarán respecto a su futuro.

Pese a que otros estudios no han encontrado relaciones o diferencias entre las dimensiones del engagement y la edad(35,36), en esta investigación se hallaron correlaciones significativas, pero baja y muy bajas entre vigor, dedicación, absorción y edad. Por esta misma línea, aunque otros estudios señalan que la dimensión con una puntuación más alta en el engagement es la absorción(37), los resultados aquí obtenidos indican que la dimensión con mayor puntuación en los trabajadores es la dedicación, seguido del vigor y por último la absorción; este comportamiento en las dimensiones ya se ha reportado(38), por lo que a nivel de intervención será menester considerar prácticas que fomenten la concentración plena en la tarea.

Ahora bien, específicamente en la dedicación, se identifica una diferencia en función de la edad y la antigüedad en la organización, siendo mayor la puntuación en el grupo de trabajadores más jóvenes y con menor permanencia en la organización. Esto puede tener una doble explicación, por un lado, el deseo de los jóvenes por mantenerse en sus primeras experiencias laborales, dada la dificultad que se registra en Colombia para acceder a un primer empleo, desde otra perspectiva, puede denotar la alta implicación que tienen los trabajadores jóvenes con las tareas que realizan en el trabajo. Así mismo, es importante que la organización de manera estratégica lidere prácticas para que el entusiasmo, orgullo e inspiración con la que los trabajadores realizan sus tareas se fortalezcan, de manera que se puedan prolongar y mantener con el paso del tiempo.

Los resultados obtenidos revelan que existe una relación significativa pero baja entre la edad del empleado y el optimismo, al respecto, en la literatura se reporta que a mayor edad mayor optimismo(20), aun cuando este hallazgo es contrario a lo reportado por otras investigaciones(17). Por otro lado, en este estudio se identifica que la edad de los empleados puede predecir el optimismo y el engagement de manera significativa $(p<0,01)$, distinto a lo reportado con anterioridad, donde se señala que no hay relación entre edad, optimismo y engagement(10). Se encontró también una relación débil entre la antigüedad y el optimismo, en la predicción se muestra que la antigüedad en la organización no prevé ninguna de las variables estudiadas, en cuanto al optimismo, estudios comprueban que no existen diferencias en el optimismo en función a la antigüedad en la organización(18). Sin embargo, respecto al engagement, resultados anteriores evidencian que los empleados que tienen más permanencia en la organización, poseen mayor compromiso hacia ésta(19).

Cabe señalar que los resultados aquí expuestos deben ser asumidos desde la particularidad cultural de la organización participante, y que basados en lo que señalan los modelos predictivos, es necesario considerar otros factores, además de las variables sociodemográficas como la edad, que si bien, juega un importante papel particularmente en el engagement, debe ser analizadas a la luz de la interacción con otros fenómenos intersubjetivos.

\section{Conclusiones}

Existe una relación significativa entre las dimensiones del engagement y el optimismo. Las correlaciones más fuertes se presentaron entre el vigor con la edad y el optimismo. Los trabajadores entre 18 y 30 años y aquellos que llevan menor antigüedad en la organización (6 a 24 meses) experimentan mayor dedicación. Por otra parte, la edad fue un predictor de las 3 dimensiones del engagement (vigor, dedicación y absorción), pero no del optimismo. La antigüedad en la organización sólo tuvo una relación muy baja con el optimismo y no se asoció con las demás variables estudiadas.

Dentro de los hallazgos, la relación más fuerte se encontró entre vigor y optimismo. Esto señala que la energía y la activación mental con la que un empleado desarrolla sus funciones, está relacionada con las creencias positivas de las personas sobre aquello que se espera en el futuro. Este hallazgo presenta una posible vía para la intervención, es decir, si las organizaciones quieren tener empleados optimistas, será necesario el fortalecimiento del vigor. 


\section{Limitaciones}

Una de las principales limitaciones de este estudio es que, al ser un muestreo no probabilístico, no se obtuvo una muestra homogénea de los grupos etarios por edad y antigüedad en la organización, asimismo, los resultados no pueden generalizarse, por tanto, las variables estudiadas podrían tener un comportamiento diferente en otros grupos poblacionales. Es fundamental comprender que, al ser constructos psicológicos, son diversos los factores (sociodemográficos, de personalidad, de grupo) que pueden afectar el curso del engagement y del optimismo, por tanto, será necesario revisar en futuros trabajos, variables personales $y$ organizacionales que moderen la relación entre estos constructos.

\section{Recomendaciones}

A partir de estos hallazgos, se sugiere a las áreas de Se sugiere a las áreas de Gestión Humana, valorar los aportes que las personas desde sus particularidades y diferencias brindan a la organización, para ello es relevante ubicar a la persona en el centro de la gestión empresarial. Sin lugar a dudas, la salud de los empleados es un propósito que debe ser incluido en las políticas organizacionales(39), se sugiere que se diseñen políticas inclusivas y de bienestar que se incorporen a la estrategia corporativa.

Finalmente, los beneficios asociados a los dos fenómenos aquí estudiados han llevado a un incremento de cursos teóricos en diversos países de América Latina; sin embargo, no se muestra la misma frecuencia en intervenciones basadas en la evidencia para gestionar esta vinculación positiva en los trabajadores. Lo anterior puede ser explicado por el déficit en la sistematización de prácticas que indiquen las mejores maneras de implementar acciones que desarrollen el engagement(40) y el optimismo(32). En este sentido, dada la relación entre vigor y optimismo, sería importante considerar en la agenda investigativa, posibles vías de actuación que promuevan los niveles de energía, resistencia mental y optimismo en los trabajadores.

Conflicto de intereses: Ninguno declarado por los autores.

\section{Referencias}

1. Meyers MC, Kooij D, Kroon B, de Reuver R, van Woerkom M. Organizational Support for Strengths Use, Work Engagement, and Contextual Performance: The Moderating Role of Age. Appl Res Qual Life. 2020;15(2):485-502. DOI: 10.1007/s11482-018-9702-4.

2. Salanova M, Llorens S, Martínez IM. Organizaciones saludables. Una mirada desde la psicología positiva. España: Aranzadi; 2019. p. 330. Disponible en: http://www.want.uji.es/wpcontent/uploads/2019/02/2019_Salanova-Llorens-yMartinez_Organizaciones-Saludables.pdf

3. Matabajoy-Montilla JM, Matabanchoy-Tulcán SM, ObandoGuerrero LM. Procesos de desarrollo del talento humano en una clínica de especialidades de Pasto, Colombia. Univ y Salud [Internet]. 2017;20(1):26-36. DOI: 10.22267/rus.182001.106.

4. Raya R, Panneerselvam S. The healthy organization construct: A review and research agenda. Indian J Occup Environ Med. 2013;17(3):89-93. DOI: 10.4103/00195278.130835.

5. Quiroz-González E, Muñoz-Jaramillo JT. Capítulo 4: El Engagement: una perspectiva desde las organizaciones saludables. En: Engagement y cambio organizacional. Editorial. Bogotá DC.: LOGOS VESTIGIUM; 2020. p. 104. Disponible en: https://repository.ucatolica.edu.co/bitstream/10983/2600 1/1/Logos\%20Vestigium\%209.pdf

6. Chiang Vega MM, Fuentealba Elgueta II, Nova Cabrera RA. Relación entre clima organizacional y engagement, en dos fundaciones sociales, sin fines dde lucro, de la región del Bio Bio. Cienc Trab [Internet]. 2017;19(59):105-12. Disponible en:

https://www.scielo.cl/scielo.php?script=sci_arttext\&pid=S0 718-24492017000200105\&lng=en\&nrm=iso\&tlng=en

7. Lisbona A, Palaci F, Salanova M, Frese M. The effects of work engagement and self-efficacy on personal initiative and performance. Psicothema [Internet]. 2018;30(1):89-96. Disponible en: http://www.psicothema.com/pdf/4455.pdf

8. Salanova M, Llorens S, Martínez IM. Aportaciones desde la psicología organizacional positiva para desarrollar organizaciones saludables y resilientes. Papeles del Psicólogo. 2016;37(3):177-84. Disponible en: https://www.redalyc.org/pdf/778/77847916004_2.pdf

9. Pérez-Chuecos RR. Engagement, autoeficacia, optimismo y situación laboral en docentes de educación básica. Rev Electrónica Investig y Docencia. 2017;(17):45-60. Disponible en: https://dialnet.unirioja.es/servlet/articulo? codigo=579754 7

10. Maury-Ortiz JG, Martínez-Lugo ME, González Colón ZL. Relación del optimismo, la personalidad resistente y el engagement con el trabajo en una muestra de empleados. Rev Puertorriquena Psicol. 2014;25(2):280-96. Disponible en: https://www.redalyc.org/pdf/2332/233245622008.pdf

11. Carver CS, Scheier MF. Dispositional optimism. Trends Cogn Sci [Internet]. 2014;18(6):293-9. DOI: 10.1016/j.tics.2014.02.003.

12. Nwanzu CL, Babalola SS. Examining psychological capital of optimism, self-efficacy and self-monitoring as predictors of attitude towards organizational change. Int J Eng Bus Manag. 2019;11:1-12. DOI: 10.1177/1847979019827149. 
13. García-Naveira A. Optimismo, Autoeficacia general y competitividad en jóvenes atletas de alto rendimiento. CCD. 2018;13(37):71-81. Disponible en: https://dialnet.unirioja.es/servlet/articulo?codigo $=643516$ 3

14. Hernández RM, Carranza RF. Felicidad, optimismo y autorrealización en estudiantes de un programa de educación superior para adultos. Interdisciplinaria. 2017;34(2):307-25. Disponible en: https://www.redalyc.org/pdf/180/18054403005.pdf

15. Levy S, Heruti I, Avitsur R. Physical injury, stress, and health: Protective role of dispositional optimism. Stress Heal. 2019;35(3):267-76. DOI: 10.1002/smi.2859.

16. Ling Suan C, Mohd Nasurdin A. Supervisor support and work engagement of hotel employees in Malaysia: Is it different for men and women? Gend Manag An Int J. 2016;31(1):2-18. DOI: 10.1108/GM-11-2014-0105.

17. De Besa Gutiérrez M, Gil Flores J, García González AJ. Psychosocial variables and academic performance related to optimism in Spanish first-year university students. Acta Colomb Psicol. 2019;22(1):164-74. Disponible en: https://actacolombianapsicologia.ucatolica.edu.co/article/v iew/1865/pdf1.1.22

18. Rojas Rodríguez M, Ramos Alvarado N. Salud, optimismo y afrontamiento en trabajadores profesionales $y$ no profesionales que trabajan en contextos altamente estresantes. Acta Colomb Psicol. 2013;16(1):149-57. Disponible en: http://www.scielo.org.co/scielo.php?script=sci_arttext\&pid =S0123-91552013000100014

19. Navarro-Abal Y, López-López MJ, Climent-Rodríguez JA. Engagement (compromiso), resiliencia y empatía en auxiliares de enfermería. Enfermería Clínica [Internet]. 2017;28(2):103-10. DOI: 10.1016/j.enfcli.2017.08.009.

20. Pedrosa I, Celis-Atenas K, Suárez-Álvarez J, García-Cueto E. Cuestionario para la evaluación del optimismo: Fiabilidad y evidencias de validez. Ter Psicológica. 2015;33(2):127-38. DOI: $10.4067 /$ S0718-48082015000200007.

21. Ato M, López JJ, Benavente A. Un sistema de clasificación de los diseños de investigación en psicología. An Psicol. 2013;29(3):1038-59. Disponible en: https://scielo.isciii.es/scielo.php?script=sci_arttext\&pid=S0 212-97282013000300043

22. Ospina C, Delgado L. Adaptación lingúística y cultural del Cuestionario Utrecht Work Engagament (UWES) a la población colombiana. Universidad Católica de Colombia. [Bogotá]: Documento no publicado; 2014.

23. Scheier MF, Carver CS. Optimism, coping, and health: assessment and implications of generalized outcome expectancies. Heal Psychol. 1985;4(3):219-47. DOI: 10.1037/0278-6133.4.3.219.

24. Sanín A, Salanova Soria M. Verificación de las condiciones psicométricas del Test de Orientación en la Vida (LOT-R) aplicado en población trabajadora Colombiana. Rev Interam Psicol Ocup. 2016;35(2):58-67. Disponible en: http://revista.cincel.com.co/index.php/RPO/article/view/1 98

25. Congreso de la República de Colombia. Ley 1090 de 2006. Por la cual se reglamenta el ejercicio de la profesión de Psicología, se dicta el Código Deontológico y otras disposiciones [Internet]. Colombia: Diario Oficial Colombia; 2006. p. 1-27. Disponible en: http://www.secretariasenado.gov.co/senado/basedoc/ley_ 1090_2006.html\#TITULO I
26. Ministerio de Salud de la República de Colombia. Resolución Número 8430 de 1993. Por la cual se establecen las normas científicas, técnicas y administrativas para la investigación en salud [Internet]. Colombia; 1993. p. 1-12. Disponible en: https://www.minsalud.gov.co/sites/rid/Lists/BibliotecaDi gital/RIDE/DE/DIJ/RESOLUCION-8430-DE-1993.PDF

27. Hair JF, Black W, Babin BJ, Anderson RE. Multivariate data analysis. Harlow: Pearson Education Limited. 2014. Disponible en: https://www.pearson.com/uk/educators/highereducation-educators/program/Hair-Multivariate-DataAnalysis-Pearson-New-International-Edition-7thEdition/PGM1052102.html

28. Aguilar Domínguez ML, De Ávila Hernandez H. Inteligencia Emocional y Engagement (Involucramiento) en el trabajo en servidores públicos con mandos medios. Universidad Nacional Autónoma de México; 2018. Disponible en: https://ru.dgb.unam.mx/handle/DGB_UNAM/TES0100078090 6

29. Ramírez Omaña DE. Prestaciones como factores de predisposición para el desarrollo del engagement en trabajadores de una organización gubernamental. Universidad Nacional Autónoma de México; 2018. Disponible en: https://ru.dgb.unam.mx/handle/DGB_UNAM/TES0100077 5894

30. Colín Flores CG. Estudio del papel mediador del engagement en el trabajo entre las demandas y recursos laborales. Psicol Iberoam [Internet]. 2018;26(2):32-44. Disponible en: http://www.redalyc.org/articulo.oa?id=133959841005\%0 APDF

31. Mayen Loaiza NC. Compromiso Laboral (Engagement) y Socialización Organizacional (Onboarding) para reducir el Síndrome del Desgaste Ocupacional en empresas. Universidad Nacional Autónoma de México; 2017. Disponible

en: https://ru.dgb.unam.mx/handle/DGB_UNAM/TES0100076593 0

32. Renaud J, Barker ET, Hendricks C, Putnick DL, Bornstein MH. The developmental origins and future implications of dispositional optimism in the transition to adulthood. Int J Behav Dev. 2019;43(3):221-30. DOI: 10.1177/0165025418820629.

33. Schönfeld F, Hess C. Asociación entre capital psicológico, engagement y rendimiento académico en estudiantes de nivel secundario de Entre Ríos. XI Congr Int Investig y Práctica Prof en Psicol XXVI Jornadas Investig XV Encuentro Investig en Psicol del MERCOSUR I Encuentro Investig Ter Ocup I Encuentro Music; 2019. 246-51. Disponible en: https://www.aacademica.org/000-111/818.pdf

34. Lu X, Xie B, Guo Y. The trickle-down of work engagement from leader to follower: The roles of optimism and selfefficacy. J Bus Res [Internet]. 2018;84:186-95. DOI: 10.1016/j.jbusres.2017.11.014.

35. Lin W, Wang L, Bamberger PA, Zhang Q, Wang H, Guo W, et al. Leading future orientations for current effectiveness: The role of engagement and supervisor coaching in linking future work self salience to job performance. J Vocat Behav [Internet]. 2016;92:145-56. DOI: 10.1016/j.jvb.2015.12.002.

36. Salas-Vallina A, Alegre J. The role of gender, age, job level and job security on physician's engagement in Spanish public hospitals. Universia Bus Rev. 2017;(56):36-51. Disponible en: https://www.redalyc.org/articulo.oa?id=43353864003 
37. Vizoso Gómez C, Arias-Gundín O. Relación entre resiliencia, optimismo y compromiso en futuros educadores. Int J Educ Res Innov. 2019;(11):33-46. Disponible en: https://www.upo.es/revistas/index.php/IJERI/article/vie $\mathrm{w} / 2938$

38. Franco-Miranda V, Quiroz-González E, Castaño-González EJ. Engagement en profesionales colombianos de la salud ¿Existen diferencias según el tipo de contrato? Rev Virtual Univ Católica del Norte. 2020;(60):159-77. Disponible en: https://www.redalyc.org/journal/1942/194263234009/ht $\mathrm{ml} /$
39. Gómez Vélez MA, Calderón PA. Salud mental en el trabajo: entre el sufrimiento en el trabajo y la organización saludable. Katharsis. 2017;(23):177-201. Disponible en: https://dialnet.unirioja.es/servlet/articulo?codigo $=588810$ 8

40. Knight C, Patterson M, Dawson J. Work engagement interventions can be effective: a systematic review. Eur J Work Organ Psychol. 2019;28(3):348-72. DOI: 10.1080/1359432X.2019.1588887. 